\title{
Effect of Electro-Acupuncture on Expression of Circadian Clock Gene Per2 and Bmal1 in Sleep Deprivation Rat
}

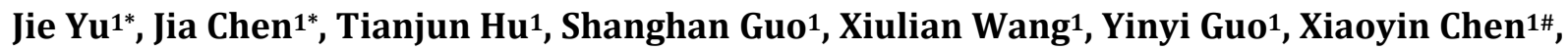 \\ Canghuan Zhao',2\# \\ ${ }^{1}$ Department of Traditional Chinese Medicine, School of Medicine, Jinan University, Guangzhou, China \\ ${ }^{2}$ Department of Acupuncture and Moxibustion, The First Affiliated Hospital of Jinan University, Guangzhou, \\ China \\ Email: "tchenxiaoyin@jnu.edu.cn," tzch@jnu.edu.cn
}

Received 24 November 2015; accepted 12 March 2016; published 15 March 2016

Copyright (C) 2016 by authors and Scientific Research Publishing Inc.

This work is licensed under the Creative Commons Attribution International License (CC BY). http://creativecommons.org/licenses/by/4.0/

cc) (i) Open Access

\section{Abstract}

Objective: The objective is to observe the treatment effect of electro-acupuncture (EA) on core circadian clock gene Per2 and Bmal1 expression in hypothalamus of sleep-deprivation (SD) rats. Methods: Thirty-two Wistar male rats were randomly divided into 4 groups. Mice in the blank control group did not receive any treatment; the remaining groups were applied with para-chlorophenylalanine (PCPA) $300 \mathrm{mg} / \mathrm{kg}$ intraperitoneal injection for 2 days. Diazepam group received intraperitoneal injection of Diazepam $(0.9 \mathrm{mg} / \mathrm{kg}$, i.p.) one time a day for 5 days, while $M$ group was treated with saline $(0.9 \mathrm{mg} / \mathrm{kg}$, i.p.) at the same time. Rats in EA group were given EA treatment, 20 minutes, once a day for 5 days, and rats in remaining groups were put into fixation-machine for the same time everyday, lasting for 5 days. Rats were sacrificed after anesthesia at the 8th day. Real-time PCR was adopted to detect the expression in clock gene Per2 and Bmal1 of each group. Results: Compared with blank control group, the expression of Per2 was significant decreased in PCPA model group $(P<0.05)$, the expression of Bmal1 was increased in PCPA model group $(P<0.05)$. Compared with PCPA model group, the expression of Per2 were significant enhanced in EA group and Diazepam group $(P<0.05)$. Simultaneously, compared with PCPA group, the expression of Bmal1 was no statistically significant in EA group and Diazepam groups $(P>$ 0.05). Conclusion: EA can significant up-regulate the expression of Per2 in SD rats, and down-regulate gene Bmal1 expression, and benefiting the weight of rats. Thus, EA is a potentially promising intervention to treat sleep-deprivation.

\footnotetext{
*Jie Yu and Jia Chen contributed equally to this work.

"Corresponding authors.
}

How to cite this paper: Yu, J., Chen, J., Hu, T.J., Guo, S.H., Wang, X.L., Guo, Y.Y., Chen, X.Y. and Zhao, C.H. (2016) Effect of Electro-Acupuncture on Expression of Circadian Clock Gene Per2 and Bmal1 in Sleep Deprivation Rat. Chinese Medicine, 7, 16-24. http://dx.doi.org/10.4236/cm.2016.71003 


\section{Keywords}

\section{Electro-Acupuncture, Sleep-Deprivation, Circadian Rhythm, Per2, Bmal1, Suprachiasmatic Nucleus (SCN)}

\section{Introduction}

As we know, sleep is a normal phenomenon which cannot be fully explained yet in biology. Sleep and awakening regulate a basic neurobiological state by homeostasis of sleep and circadian rhymes cycle (approximately 24 hours) [1]. Modern medical research showed that sleep disorders associated with the change of the circadian rhythm [2]. Acupuncture is widely used in insomnia clinically and empirically [3]. However, the potential neural mechanism underlying the therapeutic effects of acupuncture remains little known. As one of the most prevalent health complaints worldwide, sleep disorder leaded to a series of physiological changes and neuroethology alteration. Researches showed that sleep disorder seriously affected the quality of life, and impacted many cognitive fields, including attention, memory, abstract ability and decision-making ability [4] [5]. There were fertile circadian clock genes played an importance role in the master of circadian clock, suprachiasmatic nucleus (SCN), which residing in the hypothalamus of the brain [6]. SD is an alternative form of acute insomnia. As a classic medication, Diazepam has been indicated with lots of side-effects such as dependence, drug-resistance, and gastrointestinal reaction.

Acupuncture as an effectively treatment in insomnia with fewer side-effect, by contrast, acupuncture treatment plays an important role in sleep disorder. The study found that acupuncture was significant improved insomnia symptoms in the experiment [7]. A summary of clinic insomnia research confirmed the clinical effects of acupuncture for insomnia [8]. The present experiment demonstrated that acupuncture was activated specific cortex regions and showed significant difference with sham acupuncture group in SD patients [9]. Previous research suggested that according to the polysomnogram recorded, acupuncture was effectively enhanced the quality of sleep [10]. In generally, SD belonged to the category of "sleepless" and "fatigue" in TCM and its diseased region was in the brain, deficiency of yin-yang disharmony [11]. Furthermore, Shenting (GV24) and Baihui (DU20) were located on top of head in anatomical structure. One research showed that acupuncture on Shenting (GV24) and Baihui (DU20) of Du meridian was significantly improved the effectiveness of acupuncture in sleep disorder patient [12]. Summary about A-B classic of acupuncture and moxibustion recorded that acupuncture on DU meridian could "tranquilize and allay excitement”, and beneficially affected insomnia [13].

At the molecular level, the functions of the core clock transcription factors Clock, bmal1 bind to E-box and activate transcription of PRY ( Per1, Per2), and CRY (Cry1, Cry2) genes from which subsequently negative feedback transcription is initiated [14] [15]. It was demonstrated that clock genes play a circadian-independent role in sleep homeostasis [16]. As core circadian clock genes, it was indicated a significant relation between Per2 and advanced sleep phase syndrome [17]. By lasting research showed that sleep deprivation decreased Bmal1, Clock and Napas2 binding to specific clock genes, and thereby influenced circadian rhythm [18].

Previous experiments were mostly about the mechanism of acupuncture which generally discussed the relevance with neurotransmitter inhibitors [19] and immunologic factors [20]. But there was little exploration to reveal the relevance with circadian clock gene. Thereby, the object of study was to reveal the potential mechanism about the effect of electro-acupuncture on expression of circadian clock Per2 and Bmal1 genes in sleep-deprivation rats.

\section{Materials and Methods}

\subsection{Animals Preparation}

Thirty-two 9-week-old Wistar male rats $(220 \pm 20$ g) were purchased from Experiment Center of Southern Medical University, License number: SCXK <Guangdong> 2011-0015, 44002100004077. Experimental animals were raised in a control environment with a temperature of $20^{\circ} \mathrm{C} \pm 1^{\circ} \mathrm{C}$, humidity of $50 \%$ in experiment center of Jinan University. The animals were housed in accordance with the guidelines for care and use of animals in scientific research-register animal facility. Experiment time is from 8 a.m. to 5 p.m. All the animals were 
feed one week before intervention. These rats were randomly divided into 4 groups by random number table method, with 8 rats in each group.

\subsection{Reagents and Consumables}

Diazepampill (batch number 1401161) were purchased from Tianjing JinYao Co., Ltd. (Tianjing, China). PCPA (para-chlorophenylalanine) (batch number H110130000) were purchased from TCI Co., Ltd. of Japan. Sodium pentobarbital (batch number 69020100) were purchased from Beijing chemistry Co., Ltd. (Beijing China). The needles $(0.25 \mathrm{~mm} \times 25 \mathrm{~mm})$ purchased from Hualun acupuncture of Suzhou Co., Ltd., Suzhou China). EA instrument (batch number G6805-2) was purchased from Qingdao XingShen instrument Co., Ltd. (Qingdao China). EP tube (2 ml) were purchased from Shanghai RuiJie biological engineering Co., Ltd. (Shanghai China)

\subsection{Groups and Intervention}

Animals $(\mathrm{n}=32)$ were randomly divided into four groups as depicted in Table 1 .

Mice in the blank control group received no treatment.

PCPA model and intervene: para-chlorophenylalanine (PCPA), a tryptophan hydroxylase inhibitor, used to establish sleep-deprivation model in rats. In our previous research, we used PCPA model method can successfully established sleep-deprivation model [20] PCPA with depletion serotonin was dissolved in physiological saline (PS) and administrated at $300 \mathrm{mg} / \mathrm{kg}$ [21] on 1st day, 2nd day. PCPA was administrated to the three intervention group: including $\mathrm{M}$ group, D group, and EA group. After that, $\mathrm{M}$ group received intraperitoneal injection with the same amount of normal saline D group were received intraperitoneal injection of Diazepam (0.9 $\mathrm{mg} / \mathrm{kg}$ i.p.) [22] once a day for 5 days, Rats in EA group were given EA treatment once' a day for 5 days, and rats in remaining groups were put into fixation-machine (restrain) for the same time everyday, lasting for 5 days. Specific intervention is shown in Table 1.

\subsection{EA Intervention on EA Group}

The acupoints of Shenting (GV24) and Baihui (DU20) in Du meridian were chosen according to document [23] as shown in Figure 1. Shenting (GV24) is located in the median of frontalis. Baihui (DU20) is located in the median of the parietal bone. The needles $(0.25 \mathrm{~mm} \times 25 \mathrm{~mm}$ needle purchased from Hualun acupuncture of Suzhou Co., Ltd., Suzhou China) were Oblique-needled into Shenting (GV24) and Baihui (DU20) for about 2 $3 \mathrm{~mm}$ respectively. Thus, EA instrument (G6805-2 were purchased from Qingdao XingShen instrument Co., Ltd., Qingdao China) were connected with Shenting (GV24) and Baihui (DU20). The progress were handled as reported previously [24], alternated strings of dense-sparse frequencies $(60 \mathrm{~Hz}$ for $1.05 \mathrm{~s}$ and $2 \mathrm{~Hz}$ for $2.85 \mathrm{~s}$ alternately) The intensity was adjusted to induce a slight twitch of the head $(\leq 1 \mathrm{~mA})$ with the entire procedure lasting 20 min. EA treatment took once a day for 5 days. All the intervention was given by the same person.

\subsection{Behavior Observation and Weight Measurement}

Rats $(\mathrm{N}=32)$ were subjected to behavior observation everyday before intervention. To observe the characteristics including the change of hair and average food intake, and recording body weight (at 1st day, 3rd day, 5th day, 7th day, 8th day), drug were administrated according to forthwith weight of rats.

Table 1. Experiment process for each group.

\begin{tabular}{ccc} 
Group & Restrain & Intervention \\
C & Yes & None \\
M & Yes & PCPA: $300 \mathrm{mg} / \mathrm{kg}$ (i.p.) $+0.9 \mathrm{mg} / \mathrm{kg}$ Saline: (i.p.) \\
D & Yes & PCPA: $300 \mathrm{mg} / \mathrm{kg}($ i.p. $)+0.9 \mathrm{mg} / \mathrm{kg}$ Diazepam: (i.p.) \\
E & Yes & PCPA: $300 \mathrm{mg} / \mathrm{kg}$ (i.p.) +20 min EA \\
\hline
\end{tabular}




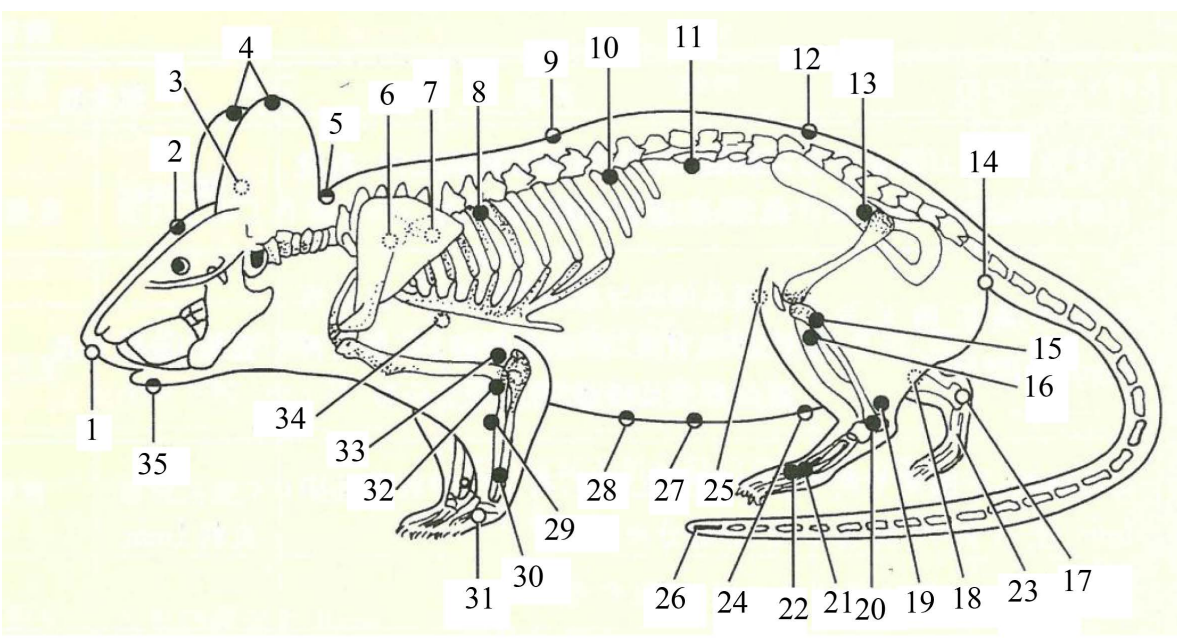

a. Point 2 is location of Shenting (GV24), Point 3 is location of Baihui (DU20)

Figure 1. Location of Shenting (GV24) and Baihui (DU20) in Rat.

\subsection{Samples Obtaining}

The rats were stopped feeding food 4 hours before sampling at the 8th day on ZT8 (16:00), the rats were sacrificed after anesthesia intraperitoneal injection of one percent sodium pentobarbital at $40 \mathrm{mg} / \mathrm{kg}$ BW. Hypothalamus were gained on the ice, washed with saline, dried, numbered different groups into EP tubes and stored at $-80^{\circ} \mathrm{C}$ refrigerator.

\section{Quantitative RT-PCR}

Gene sequence of Per2 and Bmal1 were retrieved from Genebank (http://www.ncbi.nlm.nih.gov/), all the DNA primers were designed and synthesized by shanghai RuiJie biological engineering Co., Ltd. (Shanghai China). ACTB was selected to be the reference gene. Sequence of primers employed for RT-PCR and their anticipate PCR size is shown in Table 2.

Use Trizol summary RNA extract reagent, extract summary RNA of hypothalamus issue and reverse-transcript and synthesis cDNA 1st chain, expand PCR outcome of Per2, Bmal1 partly and measure mRNA content. Total RNA was isolated from 3 - 5 inflorescence samples using RNaEXTM Total RNA Isolation Solution (Generay, China). cDNA was synthesized from $4 \mu \mathrm{g}$ total RNA using reverse transcriptase (Aidlab, China) and qRT-PCR analyses were performed on an ABI PRISM 7500 Real-Time PCR System (Applied Biosystems, USA). Specific process was following the document [25].

\section{Statistically Analysis}

All data were analyzed by using SPSS13.0 software. Quantitative data are expressed as the mean \pm standard deviation $(X \pm S)$. The single factor analysis of variance of completely randomized design was used for parameter comparison between groups. $P<0.05$ was considered statistically significant.

\section{Results}

\subsection{EA Treatment Changes the Expression of Per2 Gene and Bmal1 Gene (Table 3)}

Compared with $\mathrm{C}$ group, the expression of Per2 were significant decreased in M model group $(P<0.05)$, the expression of Bmal1 were increased in PCPA model group $(P<0.05)$. Compared with PCPA model group, expression of Per2 were significant enhanced in EA group and Diazepam group $(P<0.05)$. Simultaneously, compared with $\mathrm{M}$ group, the expression of Bmal1 were no statistically significant in EA group and Diazepam group $(P>0.05)$. Compared with D group, EA were no statistical differences in the expression of Per2 and Bmal1 genes. 


\subsection{Effect of EA Treatment on Behavior and Incremental Value of Weight}

After 2 days PCPA intervention, compared with blank control group, rats in other 3 group showed Shaggy hair, decreased feed intake. Furthermore, incremental of weight was significant decreased on 3rd day in M group, D group and EA group $(P<0.01)$. These evidences coincide with the previous reports, thus proving successful model [20]. On the 5th day and 8th day, weight of rats was increased in all groups, no intergroup statistical difference $(P>0.05)$. On the 7th day, compared with model group, other 3 groups showed significant enhanced in incremental of weight. On incremental weight in total, compared with Diazepam group, EA group were increased $(P<0.05)$. The results are shown in Table 4.

\section{Discussion}

Lots of circadian clock regulate sleep and awakening in circadian rhyme, residing in our brain. Scientist think that the suprachiasmatic nucleus (SCN), residing in the hypothalamus of the brain, programs the body to follow a 24-hour rhythm. Every cell in our body named molecular clock drive our body circadian rhythm, as well as molecular clock in SCN. SCN is an endogenous mechanism drive circadian rhythm [26]. Circadian clock is derived from positive and negative transcription feedback loops. They combine to drive circadian rhythm. In the positive feedback loop, Clock gene combined Bmal1 gene, to be transcription factor as heterodimers, induce transcription of Per and Cry genes at the E-box element include their promoters, and auto-inhibit of Per and Cry genes expression by their protein products PER and CRY. In the negative feedback loop, it is benefited by the

Table 2. Sequence of primer employed for RT-PCR and their anticipated PCR product size.

\begin{tabular}{cccc}
\hline Gene & primer & Oligonucleotide sequence 5' -3' $^{\prime}$ & length (bp) \\
\hline Actb & Front & CCTAGACTTCGAGCAAGAGA & $139 \mathrm{bp}$ \\
& Rear & GGAAGGAAGGCTGGAAGA & $101 \mathrm{bp}$ \\
Fer2 & Front & AGCCTCCTTGCTCCTACCA & $104 \mathrm{bp}$ \\
& Rear & GGATCTCGCACTTTCTTTTCA \\
\hline
\end{tabular}

Table 3. The expression of Per2 mRNAm, Bmal1 mRNA in different treatment groups $(\bar{x} \pm s)$.

\begin{tabular}{ccc}
\hline Group & Per2 mRNA & Bmal1 mRNA \\
\hline C & $1.00 \pm 0.00$ & $1.00 \pm 0.00$ \\
M & $0.45 \pm 0.12^{-}$ & $1.34 \pm 0.17$ \\
D & $0.70 \pm 0.20^{-4}$ & $1.09 \pm 0.15$ \\
E & $0.79 \pm 0.12^{-4}$ & $1.06 \pm 0.12$ \\
\hline
\end{tabular}

C is blank group, M is model group, D is Diazepam group, and EA is electro-acupuncture treatment group. ${ }^{\boldsymbol{\Lambda}} P<0.01$ vs. Model group; ${ }^{\mathbf{D}} P<0.05$ vs. blank group.

Table 4. Value of increased weight in each group.

\begin{tabular}{|c|c|c|c|c|c|c|}
\hline Group & $\mathrm{N}$ & 3d (g) & $5 d(g)$ & 7d (g) & 8d (g) & TOTAL (g) \\
\hline $\mathrm{C}$ & 8 & $8.12 \pm 1.83$ & $9.33 \pm 3.76$ & $11.28 \pm 2.68^{\wedge}$ & $5.22 \pm 3.11$ & $33.95 \pm 6.93$ \\
\hline $\mathrm{M}$ & 8 & $-11.26 \pm 7.40^{\mathbf{m}}$ & $6.62 \pm 4.97$ & $1.22 \pm 3.94$ & $2.99 \pm 2.04$ & $-0.43 \pm 3.78^{\square}$ \\
\hline $\mathrm{D}$ & 8 & $-14.51 \pm 8.57^{\square}$ & $8.19 \pm 5.88$ & $10.28 \pm 3.01^{\wedge}$ & $3.82 \pm 3.41$ & $7.78 \pm 9.24^{\star}$ \\
\hline EA & 8 & $-16.32 \pm 6.22^{\mathbf{m}}$ & $10.43 \pm 3.09$ & $14.66 \pm 5.92^{\wedge}$ & $5.33 \pm 2.84$ & $14.10 \pm 1.57^{\star \star}$ \\
\hline
\end{tabular}


reaction of Per gene combined with Cry gene, inhibit Clock gene and Bmal1 gene transcription. Thus, basic on the transcription and translation, auto-regulation feedback loop have been formed of relatively conservation way in vivo [27] [28] Per2 gene, as a negative feedback factor in circadian clock [29]. In addition to, previous research on sleep-deprivation showed that, it could induce neuron activity in the SCN [30]. A research showed that 6 hours sleep deprived could induce the expression of Per2 gene [31]. Therefore, in this study, we focused on the change in the expression of Per2. In clinic, acupuncture, as know as an efficient therapy for the sleep disorder. According to our results show that, the expression of Per2 were decreased in sleep deprived rats, and up-regulated to the normal level (approximately the blank control group) in EA group. The result showed that EA treatment may regulate the expression of Per2 gene to adjust circadian rhythm, with the underlying mechanism to be further studies.

In present study, Per2 might take part in the limbic system; it has the double action of regulating emotion and visceral function. Degrade Per1 and Per2 could reduce intestinal activity in mice [32]. According to our results, on 3rd day, compared with blank control group, model group, EA group and Diazepam group showed significant decrease in weight. The weight rebound after EA treatment and Diazepam treatment. Compared with Diazepam group, EA group showed statistical differences. Therefore EA treatment is superior in rebound weight, and may infer that EA is not merely adjusting sleep disorder but also benefit digestive system simultaneously, EA treatment shows advantage effect over Diazepam treatment. The potential mechanism is associated with the expression of Per2.

As a positive feedback factor of circadian clock, Bmal1 gene, regulating circadian rhythm, and specific combining with Clock gene as heterodimers, and inducing transcription of Per gene to form circadian rhythm [33]. A research suggested that melatonin was associated with the expression of bmal1 [34]. Furthermore, previous research showed that expression of bmal1 directly rhythmic synthesis of histamine, and regulated sleep-awakening with the circadian clock cycle [35]. According to those experiment we concluded that the expression of Bmal1 play an importance role in regulate sleep disorder. Simultaneously, scientist found that it has additional mechanisms after transcription in regulate the circadian clock [36]. According to our result, compared with blank control group, the expression of bmal1 increased definitely in model group. However, after EA treatment, the expression of Bmal1 gene showed downward tendency without statistically significant. According to our result and previous experiment, we suggest that EA treatment may not related with the expression of bmal1 gene directly. lHowever, According to the results of Table 4, compared with D group, EA showed a little advantage over D group but no statistical differences in the expression of per2 and bmal1 genes.

In recent years many research focused on gene transcription level, nevertheless more and more evidences indicated that post-translational modification play a significant role to control the stability of protein, and thereby influence many clock component [37] In post-translational level, such as microRNAs, phosphorylation [38][40], ubiquitination [41] and SUMOylation [42] have been demonstrated to regulate the circadian clock. MicroRNAs219 (miR-219) was proved in regulating the length of the circadian rhythm. [43] Recent research demonstrated that miR-219 was positive modulators of clock-and bmal1 dependent Per transcription and the expression of miR-219 is regulated by bmal1 [44]. MiR-219 may play crucial roles in modulating the circadian clock. We suspect that the mechanism of EA treatment may take multilevel regulation in circadian clock gene by further research.

\section{Conclusion}

Our study proved that EA could significantly up-regulate the expression of Per2 in SD rats, and down-regulate and the expression of Bmal1, and benefit the weight of rats. It may regulate circadian rhythm through changing circadian clock gene (Per2, Bmal1) in vivo. In regulating the function of digestive system, EA treatment is better than diazepam treatment. We speculate that EA may associate to regulate circadian rhythm by inducing Per2 gene in circadian clock mechanism.

\section{Acknowledgements}

This study was funded by Doctoral Fund of the Ministry of Education 2010 (No. 20104401110003), National Natural Science Foundation of China (No. 81273616 and 81473557), and Guangdong Natural Science Foundation (No. S2013010013434), Science and Technology Program of Guangzhou, China (No. 2014J4100106), Science and Technology Program of Guangdong, China (No. 2014A020212221). 


\section{Author's Contributions}

JY, CZ and XC conceived the study, JC, TH, SG, XW, and YG searched the databases, performed the experiment and wrote the manuscript. All authors read and approved the final manuscript.

\section{Competing Interests}

The authors declare that they have no competing interests.

\section{References}

[1] Li, J. (2012) Molecular Mechanisms of Sleep Research Progress. Journal of International Neurology and Neurosurgery, 3, 295-297

[2] Yu, H.Y. and Zhe, C. (1999) The Latest Research on Sleep Disorder Caused by Depression. Foreign Medical Sciences (Section of Psychiatry), 3, 228-317. http://dx.doi.org/10.13479/j.cnki.jip.1999.03.003

[3] Hu, J. and Sun, N.Y. (2010) Clinical Summary on Acupuncture in the Patient of Insomnia. Gansu Journal of TCM, 23, 78-80.

[4] Diekelmann, S. and Born, J. (2010) The Memory Function of Sleep. Nature Reviews Neuroscience, 11, 114-126. http://dx.doi.org/10.1038/nrn2762

[5] Goel, N., Rao, H., Durmer, J.S., et al. (2009) Neurocognitive Consequences of Sleep Deprivation. Seminars in Neurology, 29, 320-339. http://dx.doi.org/10.1055/s-0029-1237117

[6] Cheng, H.Y. and Obrietan, K. (2007) Revealing a Role of MicroRNAs in the Regulation of the Biological Clock. Cell Cycle, 6, 3034-3038. http://dx.doi.org/10.4161/cc.6.24.5106

[7] Gao, X., Wang, P., Ren, S., et al. (2013) Curative Effect of Acupuncture and Moxibustion on Insomnia: A Randomized Clinical Trial. Journal of Traditional Chinese Medicine, 33, 428-432. http://dx.doi.org/10.1016/S0254-6272(13)60143-0

[8] Tang, Y., Zhang, L.X., Hu, Y.P., et al. (2013) Clinical Research of Present Situation on Acupuncture Treatment for Insomnia in Recent 5 Years. Journal of Liaoning University of TCM, 15, 143-145.

[9] Gao, L., Zhang, M., Gong, H.H., et al. (2014) Differential Activation Patterns of fMRI in Sleep-Deprivation Brain: Restoring Effects of Acupuncture. BMC Neuroscience, 8, 1471-1482.

[10] Hu, J. (2011) The Effect of Treatment of Acupuncture Was Evaluated by Polysomnogram in Patients with Insomnia. Journal of Modern Electrophysiology, 18, 220-222.

[11] Cao, H., Pan, X., Li, H., et al. (2009) Acupuncture for Treatment of Insomnia: A Systematic Review of Randomized Controlled Trials. Journal of Alternative and Complementary Medicine, 15, 1171-1186. http://dx.doi.org/10.1089/acm.2009.0041

[12] Wang, J. and Jiang, J.F. (2006) Clinical Observation on Governor Vessel Daoqi Method for Treatment of Dyssomnia in the Patient of Depression. Chinese Acupuncture \& Moxibustion, 26, 328-330.

[13] Zhao, Y.X. (2010) Summary of the Treatment of Insomnia in A-B Classic of Acupuncture and Moxibustion. Chinese Journal of Ethnomedicine and Ethnopharmacy, 39, 39-40.

[14] Ko, C.H. and Takahashi, J.S. (2006) Molecular Components of the Mammalian Circadian Clock. Human Molecular Genetics, 15, R271-R277. http://dx.doi.org/10.1093/hmg/ddl207

[15] Ripperger, J.A. and Schibler, U. (2006) Rhythmic CLOCK-BMAL1 Binding to Multiple E-Box Motifs Drives Circadian Dbp Transcription and Chromatin Transitions. Nature Genetics, 38, 369-374. http://dx.doi.org/10.1038/ng1738

[16] Franken, P. and Dijk, D.J. (2009) Circadian Clock Genes and Sleep Homeostasis. European Journal of Neuroscience, 29, 1820-1829. http://dx.doi.org/10.1111/j.1460-9568.2009.06723.x

[17] Bae, K., Jones, C.R., He, Y., et al. (2001) An hPer2 Phosphorylation Site Mutation in Familial Advanced Sleep Phase Syndrome Drome. Science, 291, 1040-1043. http://dx.doi.org/10.1126/science.1057499

[18] Mongrain, V., La Spada, F., Curie, T. and Franken, P. (2011) Sleep Loss Reduces the DNA-Binding of BMAL1, CLOCK, and NPAS2 to Specific Clock Genes in the Mouse Cerebral Cortex. PLoS ONE, 6, e26622. http://dx.doi.org/10.1371/journal.pone.0026622

[19] Liu, Z.L., Tang, C.L., Yu, M. and Hou, Y.X. (2011) Effect of Different Intensities of Electroacupuncture on the Expression of GABA and GABRAI in Hypothalamus of Insomnia Rats by PCPA. Life Science Research, 15, $236-240$.

[20] Zhao, C.H., Li, R. and Song, Y. (2008) Effect of Electro-Aupuncture with Different Acupoint Group on IL-1, TNFand IL-6 in the Hypothalamus of Insomnia Rats. Journal of Jinan University, 29, 178-183.

[21] Koe, B.K. and Weissman, A.M. (1966) P-Chlorophenylalanine: A Specific Depletor of Brain Serotonin. Journal of 
Pharmacology and Experimental Therapeutics, 154, 499-516.

[22] Guo, S.H., Lin, Y.L., Zhao, C.H. and He, H.X. (2015) The Effect of Electro-Acupuncture on Expression of MicroRNA-132 in Sleep-Deprived Rats' Hypothalamus. Journal of Jinan University (Nature Science \& Medicine Edition), 36, 313-318.

[23] Li, Z.R., et al. (2007) Experimental Acupuncture. 2nd Edition, China Press of Traditional Chinese Medicine, Beijing, 255-257.

[24] Kim, J.H., Min, B.I., Na, H.S., et al. (2004) Relieving Effects of Electroacupuncture on Mechanical Allodynia in Neuropathic Pain Model of Inferior Caudal Trunk Injury in Rat: Mediation by Spinal Opioid Receptors. Brain Research, 998, 230-236. http://dx.doi.org/10.1016/j.brainres.2003.11.045

[25] Wang, Z.J., Liu, W. and Wang, J.J. (2014) Improving Effects of Electroacupuncture on Regenerative Feedback Loop of Superchiasmatic Nucleus in Mice with Breast Cancer. Journal of Chengdu University of TCM, 37, 20-23.

[26] Li, J.C. and Yu, D. (2004) New Process of Circadian Clock Gene. Hereditas, 26, 89-96.

[27] Jin, X., Shearman, L.P., Weaver, D.R., et al. (1999) A Molecular Mechanism Regulating Rhythm Output from the Suprachiasmatic Circadian Clock. Cell, 96, 57-68. http://dx.doi.org/10.1016/S0092-8674(00)80959-9

[28] King, D.P. and Takahashi, J.S. (2000) Molecular Genetics of Circadian Rhythms in Mammals. Annual Review of Neuroscience, 23, 713-742. http://dx.doi.org/10.1146/annurev.neuro.23.1.713

[29] Bea, K., Jin, X., Maywood, E.S., et al. (2001) Differential Functions of mPerl, mPer2, and mPer3 in the SCN Circadian Clock. Neuron, 30, 525-536. http://dx.doi.org/10.1016/S0896-6273(01)00302-6

[30] Deboer, T., Detari, L. and Meijer, J.H. (2007) Long Term Effects of Sleep Deprivation on the Mammalian Circadian Pacemaker. Sleep, 30, 257-262.

[31] Franken, P., Thomason, R., Heller, H.C., et al. (2007) A Non-Circadian Role for Clock-Genes in Sleep Homeostasis: A Strain Comparison. BMC Neuroscience, 8, 87. http://dx.doi.org/10.1186/1471-2202-8-87

[32] Hoogerwerf, W.A., Shahinian, V.B., Cornélissen, G., et al. (2010) Rhythms Changes in Colonic Motility Are Regulated by Period Genes. American Journal of Physiology—Gastrointestinal and Liver Physiology, 298, 143-150. http://dx.doi.org/10.1152/ajpgi.00402.2009

[33] Dunlap, J.C. (1999) Molecular Bases for Circadian Clocks. Cell, 96, 271-290. http://dx.doi.org/10.1016/S0092-8674(00)80566-8

[34] Innominato, P.F., Lim, A.S., Palesh, O., et al. (2016) The Effect of Melatonin on Sleep and Quality of Life in Patients with Advanced Breast Cancer. Supportive Care in Cancer, 24, 1097-1105. http://dx.doi.org/10.1007/s00520-015-2883-6

[35] Yu, X., Zecharia, A., Zhang, Z., et al. (2014) Circadian Factor BMAL1 in Histaminergic Neurons Regulates Sleep Architecture. Current Biology, 24, 2838-2844. http://dx.doi.org/10.1016/j.cub.2014.10.019

[36] Kramer, C., Loros, J.J., Dunlap, J.C., et al. (2003) Role for Antisense RNA in Regulating Circadian Clock Function in Neurospora Crassa. Nature, 421, 948-952. http://dx.doi.org/10.1038/nature01427

[37] Cheng, H.Y. and Obrietan, K. (2007) Revealing a Role of microRNAs in the Regulation of the Biological Clock. Cell Cycle, 6, 3024-3035. http://dx.doi.org/10.4161/cc.6.24.5106

[38] Kondratov, R.V., Kondratova, A.A., Lee, C., et al. (2006) Post-Translational Regulation of Circadian Transcriptional CLOCK(NPAS2)/BMAL1 Complex by CRYPTOCHROMES. Cell Cycle, 5, 890-895. http://dx.doi.org/10.4161/cc.5.8.2684

[39] Lee, B., Almad, A., Butcher, G.Q., et al. (2007) Modulates the Phase-Delaying Effects of Light in the Mammalian Circadian Clock. European Journal of Neuroscience, 26, 451-462. http://dx.doi.org/10.1111/j.1460-9568.2007.05664.x

[40] Eide, E.J., Woolf, M.F., Kang, H., et al. (2005) Control of Mammalian Circadian Rhythm by CKI Epsilon-Regulated Proteasome-Mediated PER2 Degradation. Molecular and Cellular Biology, 25, 2795-2807. http://dx.doi.org/10.1128/MCB.25.7.2795-2807.2005

[41] Busino, L., Bassermann, F., Maiolica, A., et al. (2007) SCFFbxl3 Controls the Oscillation of the Circadian Clock by Directing the Degradation of Cryptochrome Proteins. Science, 316, 900-904. http://dx.doi.org/10.1126/science.1141194

[42] Cardone, L., Hirayama, J. and Giordano, F. (2005) Circadian Clock Control by SUMOylation of BMAL1. Science, 5739, 1390-1394. http://dx.doi.org/10.1126/science.1110689

[43] Cheng, H.Y., Papp, J.W., Varlamova, O., et al. (2007) MicroRNA Modulation of Circadian—Clock Period and Entrainment. Neuron, 54, 813-829. http://dx.doi.org/10.1016/j.neuron.2007.05.017

[44] Liu, K. and Wang, R. (2012) mircroRNA-Mediated Regulation in the Mammalian Circadian Rhythm. Journal of Theoretical Biology, 7, 103-110. http://dx.doi.org/10.1016/j.jtbi.2012.03.037 


\section{Abbreviations}

EA: Electroacupuncture

Per2: Period circadian clock 2

Bmal1: Brain and muscle ARNT-like-1

SD: Sleep-deprivation

PCPA: para-chlorophenylalanine

SCN: suprachiasmatic nucleus

ACTB: $\beta$-Actin 